\title{
Change of Corrosion Potential Fluctuations with Pits Growing on Aluminum Surface
}

\author{
Shin-ichi Magaino* \\ *Industrial Research Institute of Kanagawa Prefecture
}

\begin{abstract}
Variation in fluctuations of corrosion potential with number of pits has been investigated for aluminum immersed in $0.05 \mathrm{M} \mathrm{NaCl}$ with $20 \mathrm{mg} / l \mathrm{Cu}^{2+}$. Wave forms and power spectral density (psd) of potential fluctuations of a specimen, which was preanodized to decrease number of pits, were compared with those of a specimen whcih was not preanodized, to begin with. Next the origin of variation in potential fluctuations with number of pits was found out by optical microscopy and acoustic emission measurement. It was found that periodic fluctuations existed in the period of initial few hours for a preanodized specimen. When the periodic fluctuations were observed, only a few pits existed, and the level of psd was remarkably high. For a preanodized specimen, time dependence of psd was correlated with that of D.C. component of the corrosion potential. The periodic fluctuations of the corrosion potential were found to be a result of periodic gas evolution from a single pit. Further, from the simplified mathematical analysis, it was shown the level of psd decreases as number of pits increases; this decrease is pronounced when number of pits is small.
\end{abstract}

\section{Introduction}

Several workers ${ }^{1) \sim 5}$ ) have been recognized that cathodic gas evolution is one of the origin of potential or current fluctuations during pitting corrosion of aluminum. However, Relation between cathodic gas evolution and potential fluctuations has not been analyzed in detail. In our previous paper ${ }^{5)}$, fluctuations of the corrosion potential have been analyzed for aluminum immersed in $0.05 \mathrm{M} \mathrm{NaCl}$ solutions with and without $20 \mathrm{mg} / l \mathrm{Cu}^{2+}$; it was found that the power of the signals filtered by the highpass and lowpass filters having their cut-off frequency at $1 \mathrm{~Hz}$ and $5 \mathrm{~Hz}$, respectively, was closely proportional to the rate of growth for the deepest pits; the power correlated with the rate of pit growth appeared to be related to cathodic gas evolution.

In this paper, we attempted to analyze the relation between cathodic gas evolution and potential fluctuations more closely, investigating variation in potential fluctuations with number of pits growing on aluminum surface. Wave forms and power spectral density of potential fluctuations of a specimen, which was preano-

\footnotetext{
* 3173, Showa-machi, Kanazawa-ku, Yokohama, 236 Japan
}

dized to decrease number of pits, were compared with those of a specimen which was not preanodized, to begin with. Next the origin of variation in potential fluctuations with number of pits was found out by optical microscopy and acoustic emission (AE) measurement. Further, a simplified mathematical interpretation of the results was proposed.

\section{Experimental Procedure}

\subsection{Specimens and Solutions}

The chemical composition of the aluminum used in this experimentation was: $\mathrm{Si}-0.08 ; \mathrm{Cu}-$ 0.004; Fe-0.32; Mn-0.004; Mg-0.000; Zn-0.002; Cr-0.002; Ni-0.010; Ti-0.049 (Weight \%) Specimens were polished with $3 / 0$ emery paper, etched in $10 \% \mathrm{NaOH}$ at $30^{\circ} \mathrm{C}$ and rinsed with distilled water. Then, some specimens were anodized at $30^{\circ} \mathrm{C}$ in $15 \%(\mathrm{w} / \mathrm{w}) \mathrm{H}_{2} \mathrm{SO}_{4}$ at a constant current of $130 \mathrm{~A} / \mathrm{m}^{2}$ for two minutes and were subsequently sealed in boiling distilled water for 30 minutes. Specimens were coated with Picein wax so that only an area to be tested was exposed to a solution. An area exposed was $1 \mathrm{~cm}^{2}$ for potential measurement only and was $0.25 \mathrm{~cm}^{2}$ for optical examination and acoustic emission (AE) measurement together 
with potential measurement.

The solutions consisted of $0.05 \mathrm{M} \mathrm{NaCl}$ and 20 $\mathrm{mg} / \mathrm{l} \mathrm{Cu}{ }^{2+}$ (as $\left.\mathrm{CuSO}_{4}\right)$. The temperature of the solutions was maintained at $30^{\circ} \mathrm{C}$ for the potential measurement only; other experiments were carried out at room temperature.

\subsection{Potential Fluctuations Measurements}

Potential fluctuations measurements were carried out with the apparatus schematically shown in Fig. 1. The basic constituent of the apparatus was similar to that described previously ${ }^{5)}$, except for the amplification factor ( $34 \mathrm{~dB}$ ).

Filtered data were sampled automatically over 10.24 seconds at 10 -millisecond intervals once in an hour. Immediately after the sampling of the filtered data, unfiltered data were sampled over 10 seconds at 100-millisecond intervals, then D.C. component of the corrosion potential was determined through averaging of the data.

The power spectral density (psd) was calculated for 1,024 points of the filtered data by the fast Fourier transform (FFT) with a Hanning window to decrease spectrum leakage.

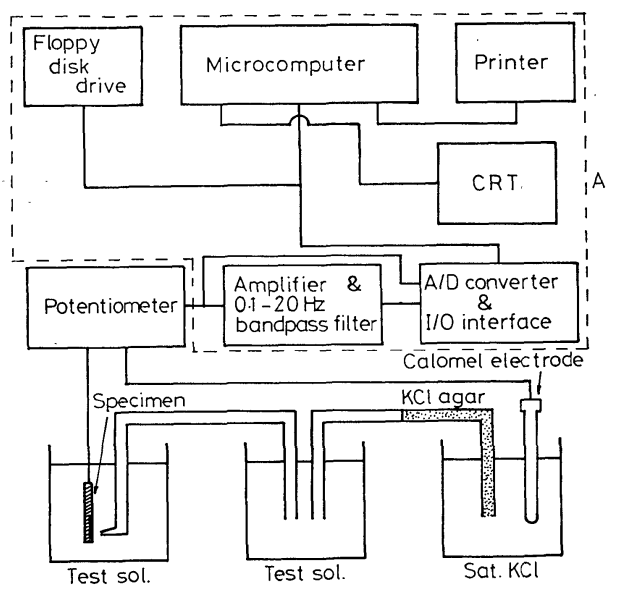

Fig. 1 Schematic diagram of the apparatus for the measurements of potential fluctuations.

\subsection{AE Measurements and Optical Examina-} tions

Acoustic emission (AE) measurements and optical examinations were carried out with the apparatus schematically shown in Fig. 2. The apparatus was connected to the system A surrounded by a dotted line in Fig. 1 .

Detected AE signals were amplified, and converted to low frequency signals; the converted AE signals and potential fluctuations were recorded simultaneously with a strip chart re-

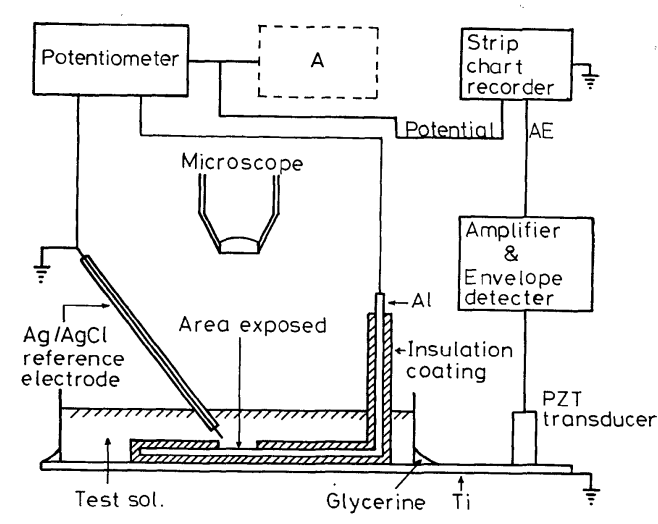

Fig. 2 Schematic diagram of the apparatus for the potential measurements, the acoustic emission measurements and optical examinations.

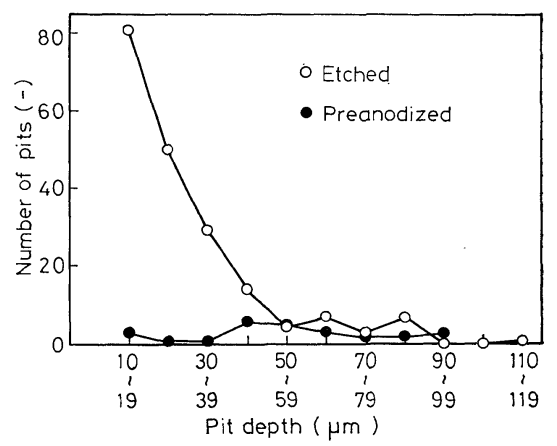

Fig. 3 Pit depth distributions on specimens with and without a preanodized oxide film: For each specimen, an area of $1 \mathrm{~cm}^{2}$ was exposed to a solution containing $0.05 \mathrm{M}$ $\mathrm{NaCl}$ and $20 \mathrm{mg} / l \mathrm{Cu}^{2+}$ for $24 \mathrm{~h}$ at $30^{\circ} \mathrm{C}$.

corder. Cathodic gas evolution was observed under a microscope $(30 \times)$, and relation between cathodic gas evolution and $\mathrm{AE}$, which was audible as a motor sound of the recorder during optical examination, was determined.

\subsection{Pit Depth Measurements}

After the specimen was tested electrochemically, the insulation coating of Picein wax was removed in toluene. Then, the specimen was immersed in conc. nitric acid containing $50 \mathrm{~g} / \mathrm{l}$ chromium trioxide to remove the corrosion products, rinsed with distilled water and dried. The depth of pits $(\geqq 10 \mu \mathrm{m})$ were measured by a calibrated microscope technique.

\section{Results}

\subsection{Pit Depth Distribution}

Pit depth distributions on specimens with and without a preanodized oxide film are shown in 
Fig. 3. For each specimen, an area of $1 \mathrm{~cm}^{2}$ was exposed to a solution containing $0.05 \mathrm{M}$ $\mathrm{NaCl}$ and $20 \mathrm{mg} / l \mathrm{Cu}^{2+}$ for 24 hours. As shown in Fig. 3, number of pits on an etched (nonpreanodized) specimen increased as pit depth decreased. On the other hand, pit depth distribution on a preanodized specimen was almost random. Total number of pits on the preanodized specimen was 26 , which was much less than that on the etched specimen (196). However, the depth of the deepest pit on the preanodized specimen $(99 \mu \mathrm{m})$ was not so different from that on the etched specimen $(112 \mu \mathrm{m})$. A shape of a pit was almost independent of existence of a preanodized oxide film.

\subsection{Wave Forms and Psd of Potential Fluc- tuations}

Examples of wave forms of the potential fluctuations obtained under the same experimental conditions as Fig. 3 are shown in Fig. 4 and Fig. 5. Wave forms for the etched specimen were almost random all through the experiment. On the other hand, for the preanodized specimen, clearly periodic fluctuations were observed in the period of initial few hours. Fig. 6 and Fig. 7 show power spectral density (psd) of the periodic fluctuations shown in Fig. 5. As shown in Fig. 6, the potential fluctuations measured after $2 \mathrm{~h}$ of immersion consisted of the signal having the fundamental frequency of $0.2 \mathrm{~Hz}$ and its higher harmonic signals. The fundamental frequency of the potential fluctua-

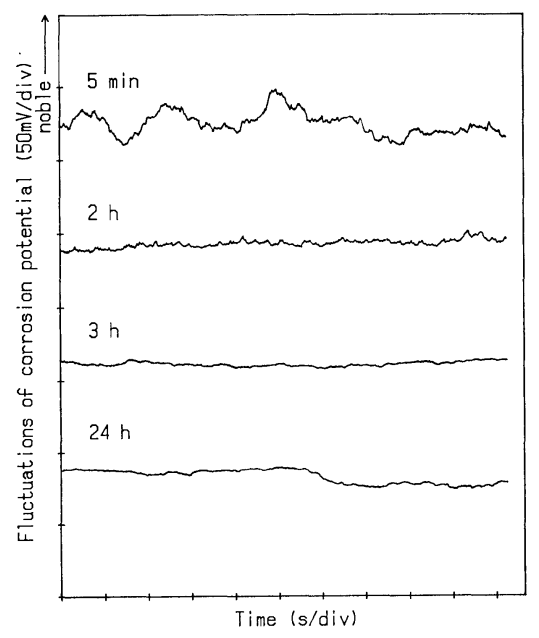

Fig. 4 Examples of wave forms of the potential fluctuations for an etched specimen: The experimental conditions were the same as Fig. 3.

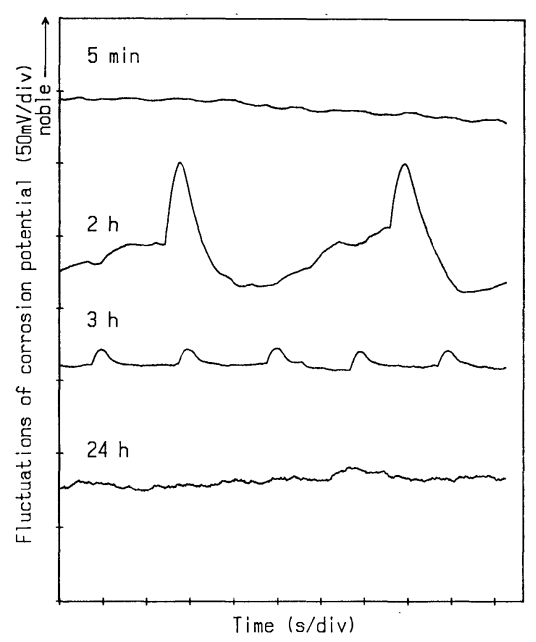

Fig. 5 Examples of wave forms of the potential fluctuations for a preanodized specimen: The experimental conditions were the same as Fig. 3.

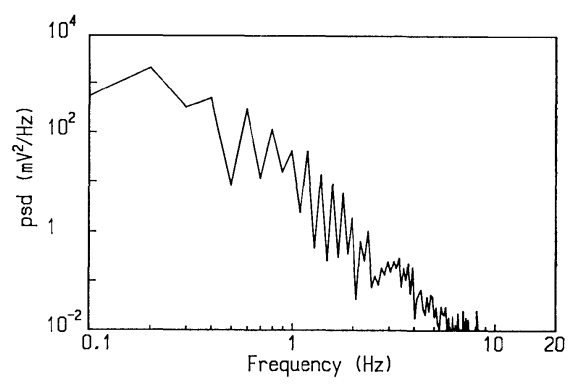

Fig. 6 Power spectral density (psd) of the periodic fluctuations shown in Fig. 5: After $2 \mathrm{~h}$ of immersion.

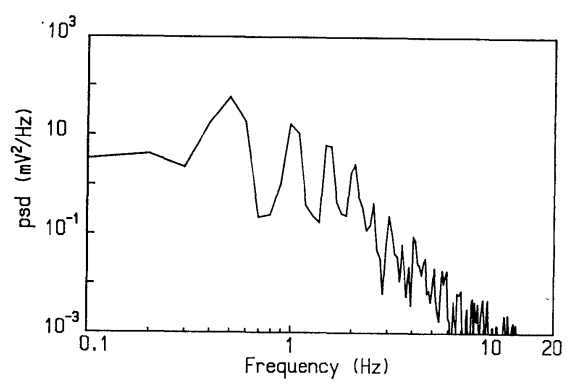

Fig. 7 Power spectral density (psd) of the periodic fluctuations shown in Fig. 5: After $3 \mathrm{~h}$ of immersion.

tions measured after $3 \mathrm{~h}$ of immersion was 0.5 $\mathrm{Hz}$ as shown in Fig. 7.

Time dependence of the levels of psd averaged in the frequency range from 1 to $5 \mathrm{~Hz}$ are 


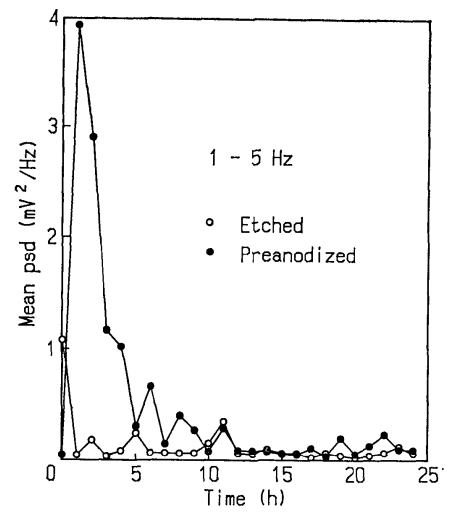

Fig. 8 Time dependence of the levels of psd averaged in the frequency range from 1 to $5 \mathrm{~Hz}$ for specimens with and without a preanodized oxide film: The experimental conditions were the same as Fig. 3.

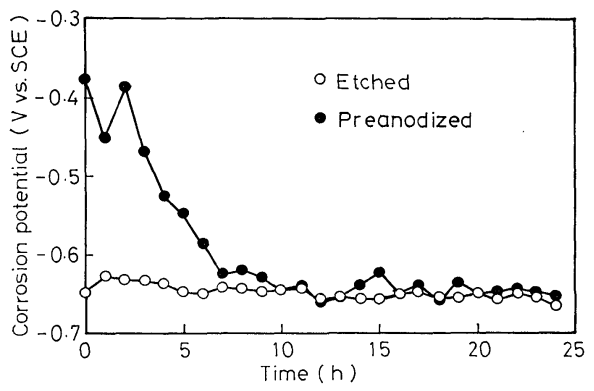

Fig. 9 Time dependence of the corrosion potential (D.C. components): The experimental conditions were the same as Fig. 3.

shown in Fig. 8. The experimental conditions were the same as Fig. 3. As shown in Fig. 8, for the preanodized specimen, the level of psd showed the remarkably high value after $1 \mathrm{~h}$ of immersion, then decreased and came close to the value for the etched specimen after about $10 \mathrm{~h}$ of immersion. Clearly periodic fluctuations were observed in the immersion period of $1 \sim 4 \mathrm{~h}$. Number of pits on a preanodized specimen immersed separately for $6 \mathrm{~h}$ was only 3 ; potential fluctuations were periodic all through the experiment. On the other hand, number of pits on an etched specimen immersed for $6 \mathrm{~h}$ was 144. Fig. 9 shows time dependence of D.C. components of the corrosion potential. As shown in Fig. 9, D.C. component for an etched specimen became a stationary value immediately after immersion. However, for a preanodized specimen, D.C. component showed a rela-

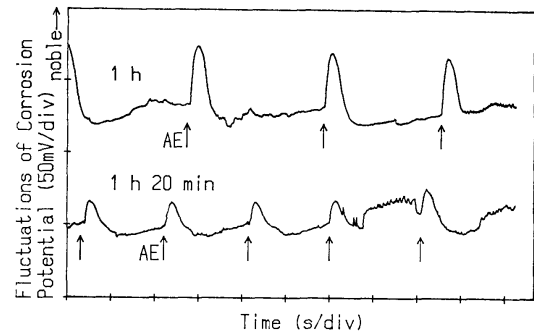

Fig. 10 Potential fluctuations and time when AE signals were detected (indicated by upward arrows): An area of $0.25 \mathrm{~cm}^{2}$ was exposed to a solution containing $0.05 \mathrm{M}$ $\mathrm{NaCl}$ and $20 \mathrm{mg} / l \mathrm{Cu}^{2+}$ at room temperature.

tively noble value immediately after immersion, shifted to a more negative value gradually as immersion period was increased, and became a stationary value after $10 \mathrm{~h}$ of immersion. Such time dependence of D.C. component for a preanodized specimen is correlated with that of psd, and presumably corresponds to increase of anodic area with immersion time.

From the results described above, it was found that the level of psd shows a remarkably high value when only a few pits are growing. The linear relationship between the rate of pit growth and the power of the potential fluctuations in the frequency range from 1 to $5 \mathrm{~Hz}$, which was shown in the previous paper ${ }^{5)}$, does not exist when number of pits varies remarkably.

\subsection{Relation between Potential Fluctuations and Gas Evolution}

Remarkably high values of psd were observed when potential fluctuations were periodic. Subsequently, origin of periodic fluctuations has been clarified through optical microscopy and acoustic emission (AE) measurement as follows:

Fig. 10 shows potential fluctuations and time when AE signals were detected (indicated by upward arrows) for a preanodized specimen $(0.25$ $\mathrm{cm}^{2}$ ) immersed in a solution containing $0.05 \mathrm{M}$ $\mathrm{NaCl}$ and $20 \mathrm{mg} / \mathrm{l} \mathrm{Cu}^{2+}$. As shown in Fig. 10, AE signals were detected when sharp fluctuations of potential were measured. From the simultaneous observation of an aluminum surface, it was found that gas evolved always when AE signals were detected. Two pits were observed on the aluminum surface, but gas evolved mainly from one of the pits. Consequently, the periodic fluctuations of the corrosion potential were found to be a result of 
periodic gas evolution from a single pit.

\section{Discussion}

We shall assume a simple pitting model to clarify the reason why the level of psd shows a remarkably high value when gas evolves from few pits. Main assumptions are as follows:

(1) It is assumed that every pit growing on an aluminum surface has the same anodic area and the same anodic current density.

(2) A main cathodic reaction is assumed to be a reduction of dissolved oxygen, and to be proceeding on an area except for pits. The proportion of hydrogen gas evolution is supposed to be smalle).

(3) An area where a cathodic reaction are proceeding is assumed to be much larger than a total anodic area.

(4) It is assumed that hydrogen bubbles evolve from all pits intermittently, but a time when a bubble evolves does not overlap.

At first we shall suppose a moment when a bubble does not evolve. At that moment a total anodic current $\left(I_{\mathrm{al}}\right)$ and a total cathodic current $\left(I_{\mathrm{cl}}\right)$ may be expressed as

$$
\begin{aligned}
& I_{\mathrm{a} 1}=n S_{\mathrm{a}} I_{0 \mathrm{a}} \exp \left(\frac{2.3}{b_{\mathrm{a}}}\left(E_{1}-E_{0 \mathrm{a}}\right)\right) \\
& I_{\mathrm{cl}}=S_{\mathrm{c}} I_{0 \mathrm{c}} \exp \left(-\frac{2.3}{b_{\mathrm{c}}}\left(E_{1}-E_{0 \mathrm{c}}\right)\right)
\end{aligned}
$$

where $E_{1}$ is corrosion potential, $n$ is number of pits, $S_{\mathrm{a}}$ is an anodic area of each pit, and $S_{\mathrm{c}}$ is a total cathodic area; $I_{0}, b$ and $E_{0}$ are exchange currents, Tafel slopes and reversible potentials, respectively, and suffixes "a" and "c" denote an anodic reaction and a cathodic reaction, respectively.

Since $I_{\mathrm{a} 1}$ must be equals $I_{\mathrm{cl}}, E_{1}$ may be determined from eqn. (1) and eqn. (2) as follows:

$$
E_{1}=\frac{b_{\mathrm{a}} b_{\mathrm{c}}}{2.3\left(b_{\mathrm{a}}+b_{\mathrm{c}}\right)} \ln \frac{S_{\mathrm{c}} I_{0 \mathrm{c}}}{n S_{\mathrm{a}} I_{0 \mathrm{a}}}+\frac{b_{\mathrm{c}} E_{0 \mathrm{a}}+b_{\mathrm{a}} E_{0 \mathrm{c}}}{b_{\mathrm{a}}+b_{\mathrm{c}}}
$$

Next we shall suppose a moment when a bubble evolves from one of the pits. At that moment ohmic drop may be generated in the pit, and corrosion current may decrease. In order to simplify the mathematical treatment, current decrease related to ohmic drop is replaced approximately by decrease of an anodic area. If $k \%$ of an anodic area in the pit is supposed to decrease, a total anodic current $\left(I_{\mathrm{a} 2}\right)$ and a total cathodic current $\left(I_{\mathrm{c} 2}\right)$ may be

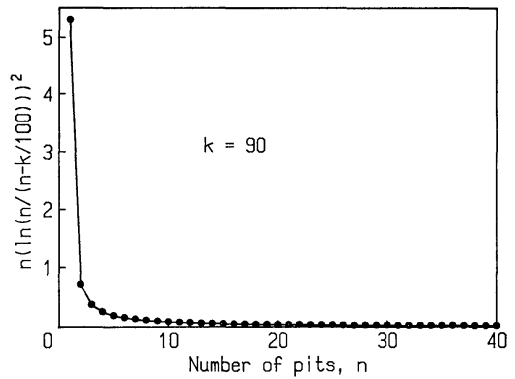

Fig. 11 Relationship between number of pits $(n)$ and $n(\ln (n /(n-k / 100)))^{2}$ in eqn. (9): $k=$ 90.

written as

$$
\begin{aligned}
& I_{\mathrm{a} 2}=(n-k / 100) S_{\mathrm{a}} I_{0 \mathrm{a}} \exp \left(\frac{2.3}{b_{\mathrm{a}}}\left(E_{2}-E_{0 \mathrm{a}}\right)\right) \\
& I_{\mathrm{c} 2}=S_{\mathrm{c}} I_{0 \mathrm{c}} \exp \left(-\frac{2.3}{b_{\mathrm{c}}}\left(E_{2}-E_{0 \mathrm{c}}\right)\right)
\end{aligned}
$$

where $E_{2}$ is corrosion potential. $E_{2}$ may be derived from eqn. (4) and eqn. (5) in a similar manner as $E_{1}$ :

$$
\begin{aligned}
E_{2}= & \frac{b_{\mathrm{a}} b_{\mathrm{c}}}{2.3\left(b_{\mathrm{a}}+b_{\mathrm{c}}\right)} \ln \frac{S_{\mathrm{c}} I_{0 \mathrm{c}}}{(n-k / 100) S_{\mathrm{a}} I_{0 \mathrm{a}}} \\
& +\frac{b_{\mathrm{c}} E_{0 \mathrm{a}}+b_{\mathrm{a}} E_{0 \mathrm{c}}}{b_{\mathrm{a}}+b_{\mathrm{c}}}
\end{aligned}
$$

Corrosion potential, which is $E_{1}$ when a bubble does not evolve, presumably shifts toward $E_{2}$ with evolution of a bubble, then returns to $E_{1}$ with release of a bubble. $E_{2}-E_{1}$ may be calculated from eqn. (3) and eqn. (6) as follows:

$$
E_{2}-E_{1}=\frac{b_{\mathrm{a}} b_{\mathrm{c}}}{2.3\left(b_{\mathrm{a}}+b_{\mathrm{c}}\right)} \ln \frac{n}{n-k / 100}
$$

Since capacitance exists in a metal/electrolyte interface, amplitude measured may be lower than $E_{2}-E_{1}$, but the amplitude must depend on $E_{2}-E_{1}$.

Let $m$ be number of bubbles evolved from each pit over a constant period. If the level of psd is assumed to be proportional to the sum of $\left(E_{2}-E_{1}\right)^{2}$, the following relation may be derived from a assumption (4) and eqn. (7):

$$
\operatorname{psd} \propto n m\left\{\frac{b_{\mathrm{a}} b_{\mathrm{c}}}{2.3\left(b_{\mathrm{a}}+b_{\mathrm{c}}\right)} \ln \frac{n}{n-k / 100}\right\}^{2}
$$

when $m, b_{a}$ and $b_{\mathrm{c}}$ are constant, eqn. (8) may be written as follows:

$$
\operatorname{psd} \propto n\left\{\ln \frac{n}{n-k / 100}\right\}^{2}
$$


Fig. 11 shows the relationship between number of pits $(n)$ and $n(\ln (n /(n-k / 100)))^{2}$ in eqn. (9) when let $k$ be 90. As shown in Fig. 11, the value of $n(\ln (n /(n-k / 100)))^{2}$ decreases as number of pits $(n)$ increases; this decrease is pronounced when number of pits $(n)$ is small. Similar relationships were obtained between $n$ and $n(\ln (n /(n-k / 100)))^{2}$ for the different values of $k$.

The mathematical result described above qualitatively agrees with the experimental result; that is, the level of psd of potential fluctuations showed a remarkably high value when gas evolves from few pits, especially from a single pit.

\section{Conclusions}

Variation in fluctuations of corrosion potential with number of pits were investigated for aluminum immersed in $0.05 \mathrm{M} \mathrm{NaCl}$ with $20 \mathrm{mg} / \mathrm{l}$ $\mathrm{Cu}^{2+}$. The primary conclusions are:

1) Periodic fluctuations of corrosion potential were observed in the period of initial few hours for a preanodized specimen.

2) When the periodic fluctuations were observed, only a few pits existed, and the level of psd was remarkably high.

3) For a preanodized specimen, time depend- ence of psd was correlated with that of D.C. component of corrosion potential.

4) The periodic fluctuations of corrosion potential were found to be a result of periodic gas evolution from a single pit.

5) From the simplified mathematical analysis, it was shown that the level of psd decreases as number of pits increases; this decrease is pronounced when number of pits is small.

\section{Acknowledgement}

The author wishes to thank Mr. H. Yamada of Industrial Research Institute of Kanagawa Prefecture for his helpful advice about acoustic emission measurement.

(Received July 30, 1985)

\section{References}

1) T. Hagyard \& J. R. Williams: Trans. Faraday Soc., 57, 2288 (1961).

2) C. B. Bargeron \& R. B. Givens: J. Electrochem. Soc., 124, 1230 (1977).

3) U. Bertocci: ibid., 127, 1931 (1980).

4) S. Magaino: Boshoku Gijutsu, 32, 712 (1983).

5) S. Magaino, M. Yasuda \& H. Yamada: ibid., 34, 157 (1985).

6) H. Kaesche: Z. Physik. Chem. N.F., 26, 138 (1960). 